\title{
A Message from the President of the American Board of Family Medicine (ABFM)
}

\author{
James C. Puffer, MD
}

(J Am Board Fam Med 2016;29:815-816.)

Feedback is critically important in helping the American Board of Family Medicine continuously improve the assessment tools that we use in our certification process. We used data in the evaluations provided after diplomates complete self-assessment activities to determine that the clinical simulations were not as useful as the knowledge assessments in helping family physicians improve their practices. This information led to our decision to no longer make the clinical simulations a mandatory part of the self-assessment and life-long learning component of continuous certification. Accordingly, we have uncoupled the clinical simulations from the knowledge assessments this past July.

This required several months of recoding the programs that drive self-assessment activity on our website, and given that we were making these major changes, we thought it time to make several other important changes in response to feedback as well. We have consistently heard that we have made the certification process too complicated and unwieldy since we introduced maintenance of certification almost 15 years ago. Before the initiation of this new paradigm, the certification process was rather straightforward. A family physician took the initial certification examination, and after passing it, he or she maintained a full, valid, and unrestricted medical license, accumulated 300 continuing medical education credits, and completed the computerized office record review-retrospectively auditing 2 charts each for 1 acute and 1 chronic medical condition-before taking the examination again 6 or 7 years later.

Although the new maintenance of certification process replaced the computerized office record

Conflict of interest: The author is president of the ABFM. review with online modules to facilitate more efficient completion of quality improvement activities and added new self-assessment modules as well, our overarching, integrated approach to continuing certification changed little. However, we complicated it with all sorts of new terminology and acronyms - Parts I, II, III, and IV, (Self-Assessment Module) SAMs, (Performance in Practice Module) PPMs, (Methods in Medicine Module) MIMMs and the like. As if that were not enough, we had multiple payment plans, and these plans have changed several times during the past 15 years so that we now have multiple permutations of payment methodology that are dependent on when a diplomate transitioned into this new process!

That all changed in July. When diplomates logged on to our website after July 21st, they noticed that all this confusing terminology has gone away. We have replaced it with language that we think more accurately reflects our continuing, integrative approach to the certification process. The activities that diplomates will complete to continue their certification have been named to accurately describe what they are. To increase flexibility to meet requirements for continuous certification, all these activities will be assigned a point value, and diplomates simply need to accumulate 50 points every 3 years with the completion of at least 1 knowledge self-assessment, at least 1 performance improvement activity and any other activities of their choosing to reach this point total. Although approximately half of our diplomates are familiar with this point system, the other half are not, since they have not yet transitioned into the continuous certification process. We have provided a detailed crosswalk for those not familiar with this process to help them effortlessly move into the new point system.

Payment has been simplified as well. The previous methodology was confusing because it always 
seemed that payment was associated with paying for a module when in fact diplomates were paying a process fee that amortized the total cost of certification (including the examination) over their entire certification cycle and which depended on whether they chose a 7- or 10-year option. Now everyone will simply pay that same amount on an annual basis. Once the annual fee has been paid, diplomates can undertake and complete as many activities as they would like. If they find that they do not like a particular module, they can simply quit and try another module that may be more to their liking at no additional cost. Given that many like to prepay their fees, we have reinstated the ability to do that as well. As previously announced, we have also implemented the $50 \%$ discount for diplomates over the age of 70 years that will be retroactive to the beginning of this calendar year.

We hope that these changes will simplify and make the certification process more efficient, but more importantly, we hope it underscores that certification is a continuous process that begins in residency training and continues throughout a family physician's professional career. This was the original intent of those that founded the Board in 1969 , and we hope to make that more explicit as we move forward. As many know, we have other initiatives under way to further simplify and reinforce this principle. This includes the recent roll out of our registry, PRIME, that many have already begun to use, as well as our recent announcement of the continuous knowledge selfassessment activity and the new performance improvement platform integrated with PRIME that we will introduce in early 2017 . We will also be announcing changes to the format of the examination and describing the data that our Board of Directors used to mandate those changes at the end of this year.

We realize that these changes will necessitate "unlearning" old terminology and acquainting oneself with new language, but we believe that the ultimate goal of simplifying the certification process will justify these changes. As always, we welcome the feedback of our diplomates; it has been critical in allowing us to continue to improve the certification process so that it is both meaningful and efficient. 